\title{
Study of the structurally sensitive physical characteristics of materials used to fabricate highly loaded elements of rolling stock
}

\author{
Yury Matyash ${ }^{1, *}$, Yury Sosnovsky ${ }^{1}$, Evgeniy Kondrikov ${ }^{1}$ and Dmitriy Muravyov ${ }^{1}$ \\ ${ }^{1}$ Omsk State Transport University, Karl Marx Ave., 35, Omsk, 644046, Russia
}

\begin{abstract}
The paper presents the results of laboratory tests of steel samples. The values of the coercive force and the results of spectral analysis of the samples under study at room temperatures are given. The dependences of the thermoelectric power, the resistivity (specific conductivity), and the Young's modulus on the number of loading cycles at a frequency of $50 \mathrm{~Hz}$ are studied. The loading amplitude did not exceed $1 \%$ of the elastic limit.
\end{abstract}

\section{Introduction}

According to the strategic direction of innovative development of the transportation process of JSC "Russian Railways", the following are the priority directions of development: introduction of heavy cargo trains (up to 18 thousand tons, 780 axles); an increase in the specific load per axle (27 tons/axle and more), and an increase in the speed (up to $120 \mathrm{~km} / \mathrm{h}$ ). At the same time, issues related to the safety and accident-free operation of the transportation process become especially important and relevant.

For the timely detection of the occurrence of various types of malfunctions of freight cars, complexes (MCTM-02, HSC, CTM, ACCS, and ASDND) are located on the approaches to maintenance points, which provide diagnostics of the technical condition of the freight rolling stock on the moving train [1]. MCTM-02 is a multifunctional complex of technical means for diagnostics of running parts of railway rolling stock. For the processing of incoming signals from KTSM-02, a hardware and software complex (HSC) is used on the basis of a laptop with special software. CTM - complex of technical measurements. ACCS - automatic coupler control system. ASDND - automatic system for detecting cars with negative dynamics.

The table shows the main units of cars, the defects of which are identified by these diagnostic complexes. Despite the fact that the introduction of these complexes is extremely necessary and relevant, they all allow determining only the critical values of the diagnosed parameters and do not allow detecting a number of defects (for example, loss of the elastic properties of the shock absorbing device of the coupler), which significantly affect the safety transportation process.

\footnotetext{
*Corresponding author: sosnovskyym@mail.ru
} 
Table 1. Typical types of defects detected by existing diagnostic complexes.

\begin{tabular}{|c|c|c|c|c|c|}
\hline \multirow{2}{*}{ Units of freight cars } & \multicolumn{4}{|c|}{ Areas of application of diagnostic complexes } \\
\cline { 2 - 6 } & MCTM-02, HSC & $\begin{array}{c}\text { DDW, CSVDL } \\
\text { (NIIAS), WILD }\end{array}$ & CTM & ACCS & ASDND \\
\hline Axlebox units & + & + & + & & \\
\hline Wheel pairs & & + & & + & \\
\hline Automatic coupler & & & & & + \\
\hline Car body and frame & & & & & + \\
\hline Brake equipment & & & & & + \\
\hline Details of car trucks & & & & & \\
\hline
\end{tabular}

In depot conditions, the most common practical methods for identifying defects are visual inspection, ultrasonic and magnetic particle methods of nondestructive examination. At the same time, the assessment of the mechanical properties of metal at repair facilities of JSC Russian Railways is not carried out at all. This is due to the lack of equipment that makes it possible to detect a change in the structure of metal, the mechanical properties and the ability to predict the behavior of the part during operation.

The magnetic particle inspection method is used to detect defects in the surface and subsurface layers of the material. Using this method, it is possible to detect defects of about $0.5 \mathrm{~mm}$ in length, $2.5 \mathrm{~mm}$ in width and more [2].

The ultrasonic method is based on recording the parameters of elastic waves that arise or are excited in the object of control. This kind of control allows determining defects in the form of cracks, pores, discontinuities, measuring the geometric parameters of the inspection object, slag and graphite inclusions, coarse granulation, as well as determining the depth of defects, coordinates, the orientation of the crack relative to the surface of the introduction of elastic vibrations, and the equivalent size of the defect [3].

Among the domestic works, special attention is paid to the paper [4] in which the authors established about twenty correlation dependences of the physical characteristics of inorganic crystals with their structural weakness: melting temperature, relative and absolute hardness, surface energy, Young's modulus, etc. At the same time, under weakness is meant a certain parameter that indirectly characterizes the energy of the interatomic interaction of inorganic crystals. It should be noted that the issue of the quick registration of the above-mentioned characteristics is still open.

In Finland, the control system of vertical forces of interaction of wheels with rails WILD [5] became widely used. This system is designed to check the condition of the wheels and to prevent defects that can affect the safety of traffic: sliders, chips, asymmetrical wheels, etc., which can cause rail and/or car damages. The module of the WILD system consists of a track panel with a length that includes two rails, sixteen sleepers, twenty strain gauges for sleepers, and twenty-two strain gauges for rails. At the end of 2015, Finland has installed and commissioned thirteen such systems.

In the USA and other countries, the technology of machine or computer vision is widely used [6, 7], based on the monitoring of car structures and their defects. Such a system makes it possible to control the position of the friction wedges as a criterion for the detach of car due to the uneven load on the side frame of the car truck, the presence or absence of brake pads, the absence of the axlebox elements (bolts, covers), the closing of the spring coils, the difference in the height of the axle beams of the coupler and much more. The development of this technology in combination with additional preventive maintenance systems has a good prospect for ensuring the safety of the railway traffic.

On the railways of the Netherlands, the Quo Vadis system for the weighing of rolling stock in motion was introduced and widely used [8].

At the same time, an analysis of the existing methods for diagnosing the residual resource showed that none of them makes it possible to estimate the dynamics of states associated 
with a change in the structure of the metal, which leads to the appearance of defects registered by existing methods.

Expansion of information about structural changes in steel products during their operation can significantly increase the accuracy and reliability of existing methods and lead to the emergence of new, most effective ways of diagnosing the residual resource. For this, it is necessary to know the relationship between the available registrable steel parameters (for example, the physical characteristics associated with the metal structure) and its mechanical properties. Analysis of the scientific literature has shown that such dependences are nearly absent or extremely rare and contradictory. The existing methods do not allow making a reliable assessment of the nature of the development of changes in the structure of the metal, which, in turn, lead to the appearance of defects registered by methods of nondestructive examination.

One of the reasons for this situation is the lack of reliable methods and appropriate equipment that allows determining and predicting the change in the structure and mechanical properties of metal units and parts during operation $[9,10]$. That is why the problem of determining the residual resource remains very relevant.

\section{Materials and methods}

The problems associated with the accumulation of fatigue damages in the process of elastic deformations of metals are quite wide and varied. It is established that the process of fatigue failure of metals consists of two periods: the initiation and propagation of fatigue cracks [11]. Taking into account the fact that the crack propagation in the metal has an explosive character [12], the authors suggested that the process of fatigue crack initiation (predefective state) is associated with structural changes in the studied metal objects and, accordingly, with the change of various structurally sensitive physical characteristics [13]. On the other hand, various semi-empirical relationships [4] connecting the energy parameters of metal products with their structural weakness are known. These dependences are characterized by a rather high degree of reliability of approximation and are not inferior to the corresponding data obtained in the framework of other energy approaches. Qualitative and quantitative confirmation of the relationship between the physical and chemical properties of metals and their performance characteristics are other results found in the scientific literature. For example, the authors of [14] established a connection between the eddy-current magnetooptical images of the surface of the main gas pipelines and the structure of the material after many years of operation. The influence of performance characteristics on the hardness of automatic couplers (embrittlement) of freight cars of railway transport has been established [15]. The work [16] shows the effect of temperature on the internal surface of distillation columns after long-term operation.

To determine the relationship with structural changes in the metal objects under study and taking into account the materials presented above, methods and tools for experimental studies were determined.

To achieve this goal, a unique laboratory facility was designed and created at OmSRTU with which it is possible to establish a connection between the physical and technical characteristics of steel ferromagnetic samples [17].

As the objects under study, steel samples in the form of a rectangular parallelepiped made of steel used for making automatic couplings, rails, etc. were fabricated at the enterprise VRK-1 Omsk (Fig. 1). 


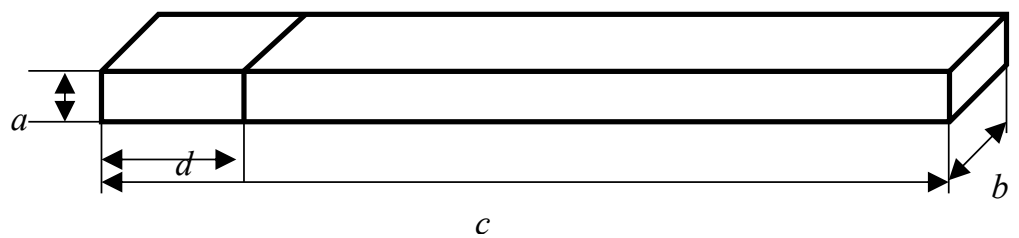

Fig. 1. Form and designation of the sample.

The test sample is rigidly fixed at one end to the fixing device (part d), and the loose end is placed in the region of the variable magnetic field, under which the free end of the sample begins to vibrate at a frequency equal to the frequency of the variable magnetic field. The nature of the load can be variable: pulsed, sinusoidal, meander, asymmetric. Through certain intervals of oscillation, a weight of a different mass is suspended from the free end of the sample. Under the influence of the load weight $\mathrm{F}$, the free end bends by the value $\Delta$ (Fig. 2). The deflection is measured by a micrometer.
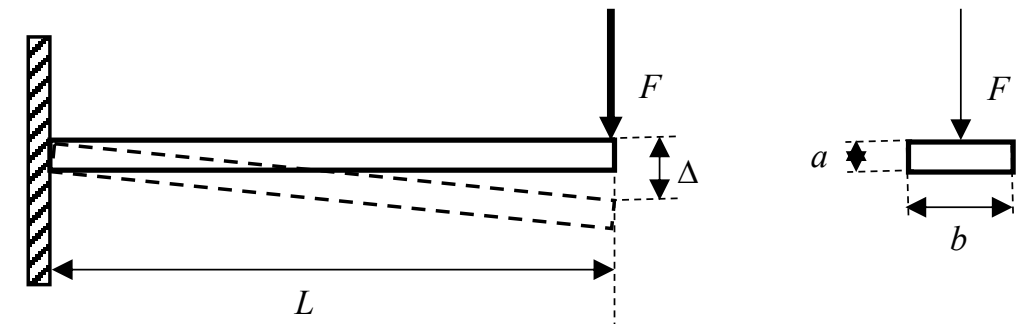

Fig. 2. Parameters for determining the Young's modulus.

By the size of the deflection and the parameters of the sample, the Young's modulus can be calculated from the formula:

$$
E=\frac{4 F L^{3}}{b a^{3} \Delta}
$$

The specific electrical resistivity ( $\rho$ ) or reciprocal to it specific electrical conductivity $\gamma$ determines the current density $(j)$ in the conductor when an external electric field with intensity (E) is applied to it (Ohm's law). To determine the specific electrical resistivity of the steel samples $\rho$ or the specific conductivity $\gamma$, a special hinged module was developed, which provided electrical contact with the sample under study. The circuit for measuring the electrical conductivity is shown in Fig. 3.

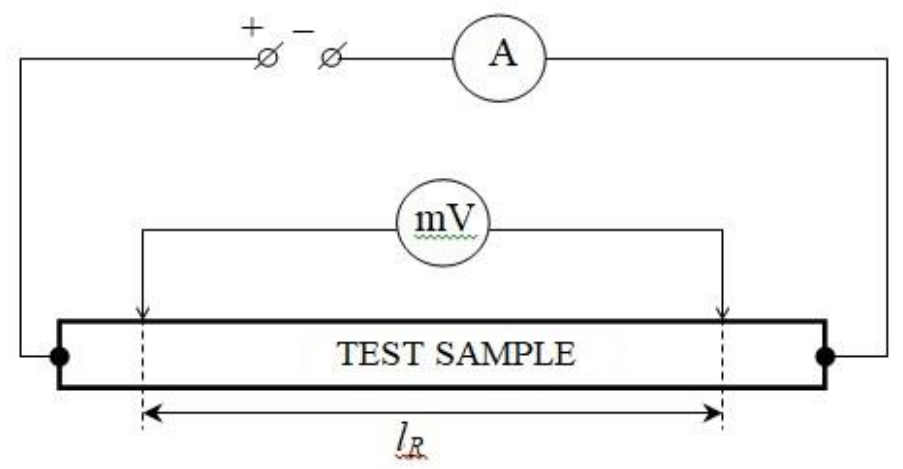

Fig. 3. Scheme for measuring the electrical conductivity of samples. 
As a voltage source, the power supply unit VS-24M was used. To calculate the specific electrical conductivity and resistivity, the following expressions are used:

$$
\gamma=\frac{I \cdot l_{R}}{U a b} \text { and } \rho=\frac{U a b}{I \cdot l_{R}}
$$

Determination of the thermoelectric power coefficient $\alpha$ is based on the Seebeck effect the appearance of the electromotive force $\varepsilon$ in a conductor in which there is a temperature gradient $\Delta \mathrm{T}: \varepsilon=\alpha \Delta \mathrm{T}$. On the other hand, the same temperature gradient determines the electromotive force $\varepsilon \mathrm{T}$, which appears in the thermocouple copper-constantan: $\varepsilon \mathrm{T}=\alpha \mathrm{T} \Delta \mathrm{T}$, where $\alpha \mathrm{T}=42.5 \mu \mathrm{V} / \mathrm{K}$ thermoelectric power coefficient of a copper-constantan thermocouple.

Taking into account the foregoing, the calculation formula for determining the thermoelectric power coefficient of the test samples is presented below:

$$
\alpha=\frac{\varepsilon}{\varepsilon_{T}} \cdot \alpha_{T}
$$

To measure the thermoelectric power coefficient of the test samples, a heater and a cooler were used. The scheme for measuring the thermoelectric power coefficient is shown in Fig. 4.

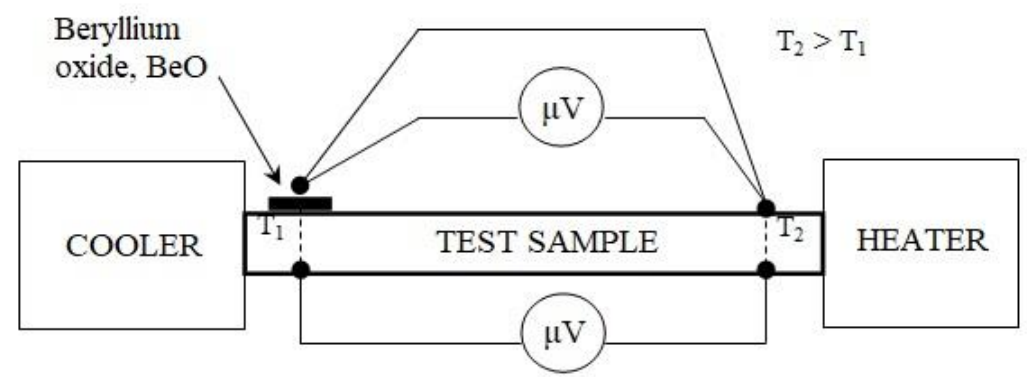

Fig. 4. Scheme for measuring the thermoelectric power coefficient.

A microvoltmeter was used to measure the thermoelectric power. Between one of the thermocouple junctions and the sample, a dielectric beryllium plate with a high thermal conductivity was installed, which made it possible to avoid short-circuiting the thermocouple.

The list of measuring instruments and certified testing equipment is given in Table 2 .

Table 2. List of measuring instruments and testing equipment.

\begin{tabular}{|c|l|c|c|c|c|}
\hline $\begin{array}{c}\text { No } \\
\mathrm{n} / \mathrm{n}\end{array}$ & \multicolumn{1}{|c|}{ Name } & Type & $\begin{array}{c}\text { Limit of } \\
\text { measurement }\end{array}$ & $\begin{array}{c}\text { Scale } \\
\text { interval }\end{array}$ & $\begin{array}{c}\text { Accuracy } \\
\text { class }\end{array}$ \\
\hline 1 & Microvolt Nano-Ammeter & F 136 & $5 \mathrm{mV}$ & $0.1 \mathrm{mV}$ & 1,5 \\
\hline 2 & Microvolt Nano-Ammeter & F 136 & $500 \mu \mathrm{V}$ & $10 \mu \mathrm{V}$ & 1,5 \\
\hline 3 & Microvolt Nano-Ammeter & F 136 & $250 \mu \mathrm{V}$ & $10 \mu \mathrm{V}$ & 1,5 \\
\hline 4 & Microvolt Nano-Ammeter & F 136 & $100 \mu \mathrm{V}$ & $2 \mu \mathrm{V}$ & 1,5 \\
\hline 5 & Microvolt Nano-Ammeter & F 136 & $50 \mu \mathrm{V}$ & $1 \mu \mathrm{V}$ & 1,5 \\
\hline 6 & Microvolt Nano-Ammeter & F 136 & $10 \mu \mathrm{V}$ & $0.2 \mu \mathrm{V}$ & 1,5 \\
\hline 7 & Ammeter & M 109 & $10 \mathrm{~A}$ & $0.1 \mathrm{~A}$ & 0,5 \\
\hline 8 & Ammeter & M 109 & $1 \mathrm{~A}$ & $0.01 \mathrm{~A}$ & 0,5 \\
\hline 9 & Micrometer & - & $5 \mathrm{~cm}$ & $0.01 \mathrm{~mm}$ & - \\
\hline 10 & Dial gauge & - & $1 \mathrm{~cm}$ & $0.01 \mathrm{~mm}$ & - \\
\hline
\end{tabular}




\section{Results}

Using the emission spectrometer ARGON-5SF, a spectral analysis of some samples was preliminary carried out in the OmSRTU research laboratory, the results of which are given in Table 3.

Table 3. Spectral analysis of some samples.

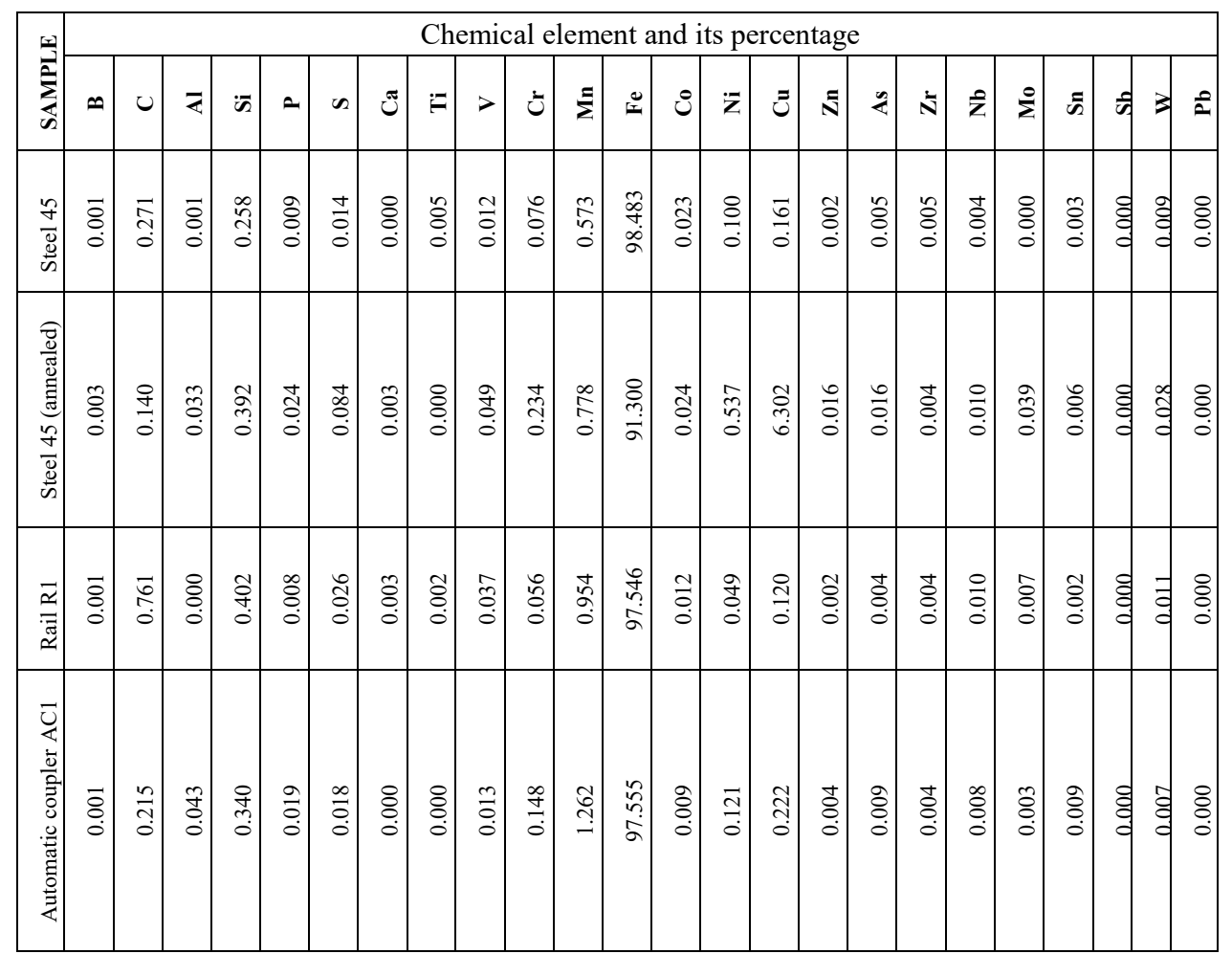

Using the K-61 structuroscope, the coercive force of all the samples used in the experiment was determined. To do this, a coercimeter was applied along the sample in two opposite directions, and then, the average result was determined according to these indications. The results of measurements are shown in Table 4.

Fig. 5 shows the dependence of the thermoelectric power of different samples on the number of loading cycles. Attention should be paid to the fact that the thermoelectric power values of the samples made from the automatic coupler of the freight car (AC1 and AC2), whose chemical and physical properties are listed in Tables 2 and 3, respectively, are significantly lower than those made of rail and steel 45 .

Fig. 6 shows the dependences of the specific resistivity of samples made of steel 45 on the number of loading cycles. Samples AN (st 45) and AN4 (st 45)2 were annealed of the first kind without phase recrystallization. It can be seen from Fig. 6 that the specific resistivity of the annealed samples has increased significantly 
Table 4. Magnetic properties of samples.

\begin{tabular}{|c|c|c|c|c|c|}
\hline \multirow{2}{*}{ No } & \multirow{2}{*}{ Sample } & \multirow{2}{*}{ Refined name } & \multicolumn{2}{|c|}{ Direction } & \multirow{2}{*}{$\begin{array}{c}\text { Coercive force, } \\
<\mathrm{Hc}>\end{array}$} \\
\hline & & & direct & opposite & \\
\hline \multirow[t]{5}{*}{1.} & \multirow{5}{*}{$\begin{array}{l}\text { Automatic } \\
\text { coupler }\end{array}$} & $\mathrm{AC} 1$ & 81 & 79 & 80,0 \\
\hline & & $\mathrm{AC2}$ & 82 & 82 & 82,0 \\
\hline & & $\mathrm{AC} 3$ & 84 & 83 & 83,5 \\
\hline & & AC4 & 89 & 87 & 88,0 \\
\hline & & AC5 & 92 & 93 & 92,5 \\
\hline \multirow[t]{5}{*}{2.} & \multirow[t]{5}{*}{ Rail } & R1 & 156 & 154 & 155,0 \\
\hline & & R2 & 163 & 159 & 161,0 \\
\hline & & R3 & 162 & 161 & 161,5 \\
\hline & & R4 & 152 & 154 & 153,0 \\
\hline & & R5 & 149 & 151 & 150,0 \\
\hline \multirow[t]{5}{*}{3.} & \multirow[t]{5}{*}{ Steel 45} & ST45-5 & 77 & 77 & 77,0 \\
\hline & & ST 45-6 & 79 & 78 & 78,5 \\
\hline & & ST 45-2 & 78 & 77 & 77,5 \\
\hline & & ST 45-3 & 74 & 75 & 74,5 \\
\hline & & ST 45-5 & 77 & 77 & 77,0 \\
\hline \multirow[t]{5}{*}{4.} & \multirow{5}{*}{$\begin{array}{ll}\begin{array}{l}\text { Steel } \\
\text { (annealed) }\end{array} & 45\end{array}$} & ST 45-AN1 & 142 & 143 & 142,5 \\
\hline & & ST 45-AN2 & 157 & 157 & 157,0 \\
\hline & & ST 45-AN4 & 141 & 143 & 142,0 \\
\hline & & ST 45-AN3 & 144 & 145 & 144,5 \\
\hline & & ST 45-AN1 & 142 & 143 & 142,5 \\
\hline
\end{tabular}

Fig. 7 shows the dependences of the Young's modulus of various samples (automatic coupler - AC-2, steel 45-5 (st. 45), rail-R2 (rail), annealed sample - AN1 (st45)) on the number of loading cycles. It can be seen that the number of load cycles does not influence the values of the Young's modulus due to the small loading amplitude.

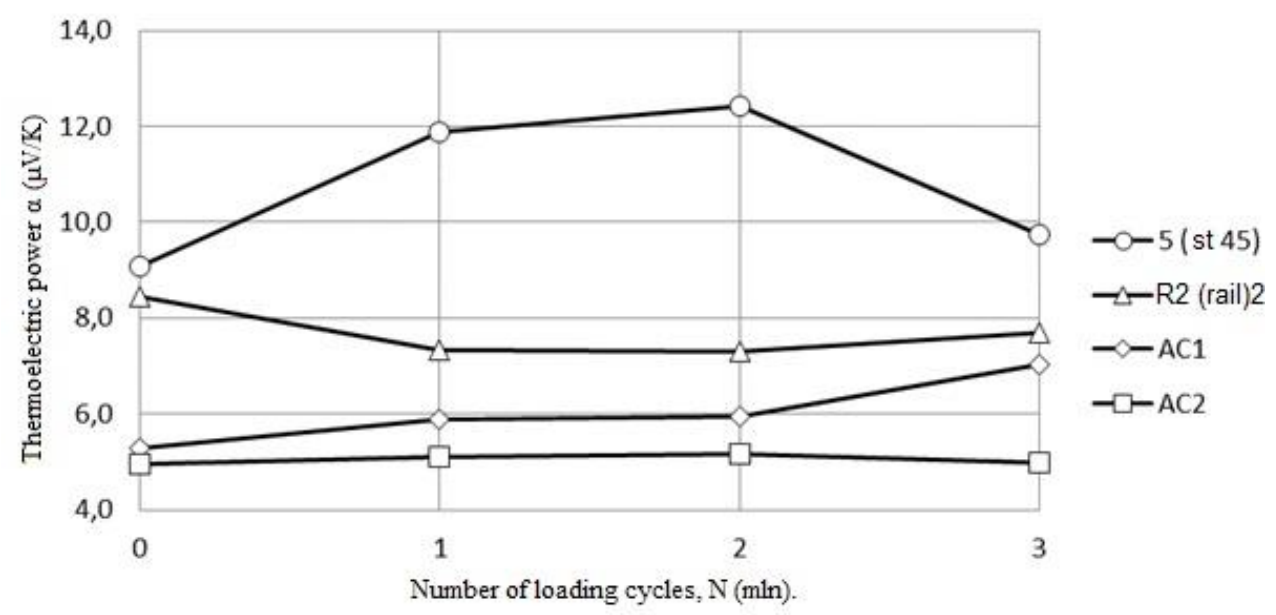

Fig. 5. Dependence of thermoelectric power of different samples on the number of loading cycles. 


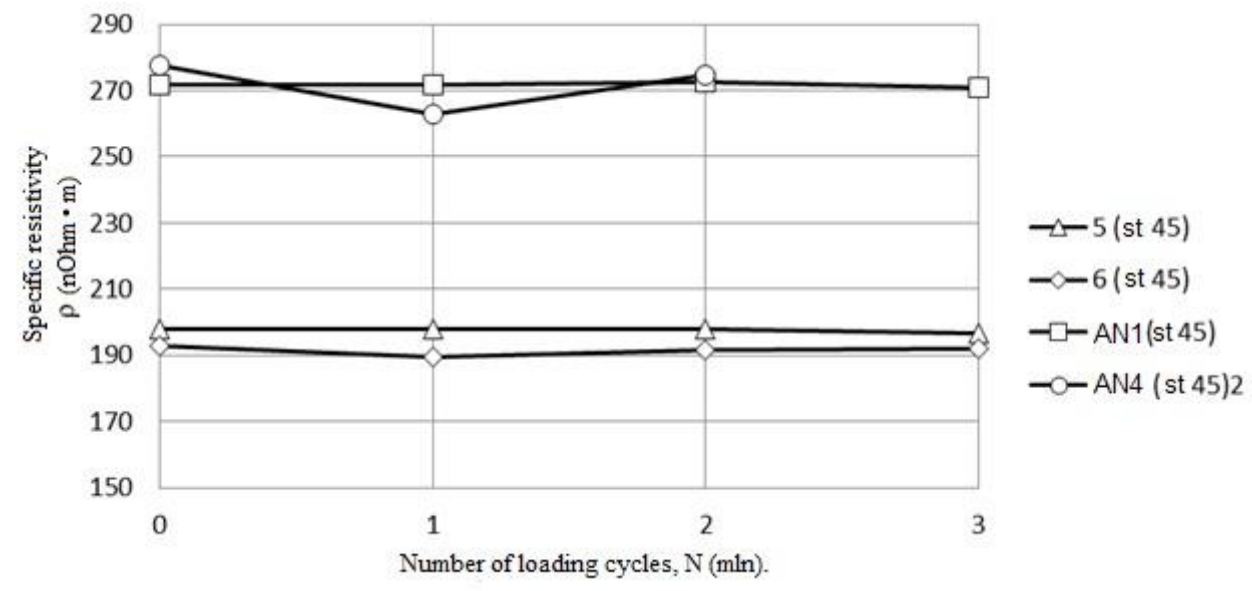

Fig. 6. Dependence of the specific resistivity of different samples on the number of loading cycles.

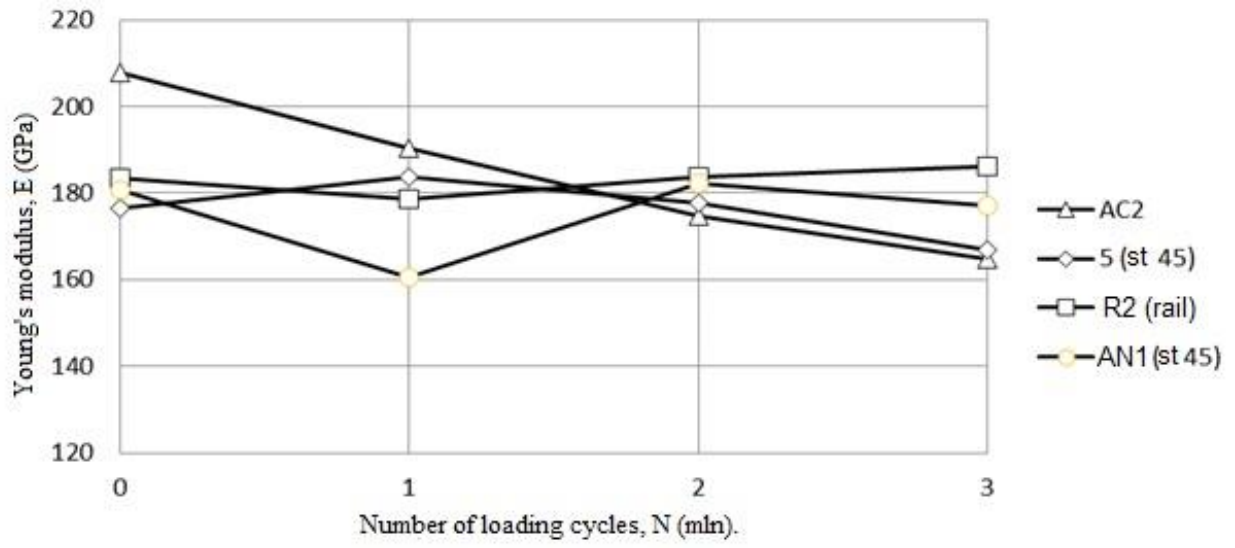

Fig. 7. Dependence of the Young's modulus of different samples on the number of loading cycles.

\section{Results}

At this stage of studying the structurally sensitive physical characteristics of materials used to fabricate highly loaded elements of rolling stock, the following results were obtained:

1. From the results presented in Fig. 5, it follows that the lowest thermoelectric power values correspond to samples of automatic coupler's materials (AC1 and AC2), which was previously subjected to embrittlement during operation [15].

2. The specific resistivity of the annealed samples of steel 45 exceeds the specific resistivity of unannealed samples of the same steel by approximately $80 \mathrm{nOhm} \cdot \mathrm{m}$, which is about $40 \%$. This allows developing a way of input control of the quality of fabricating highly loaded units and parts of the railway transport.

3. The results of measuring the Young's modulus are useful for predicting the residual resource, since they can give an idea of the degree of change in the mechanical characteristics of the objects under study.

4. The value of the coercive force depends on the domain structure of the object under study (see Table 4) and its mechanical properties [14]. Consequently, the degree of increase in 
the external magnetic field is a structural characteristic of the surface under study, which makes it possible to increase the reliability of the determination of the residual resource.

5. The values of thermoelectric power, specific resistivity, and the Young's modulus do not depend on the number of oscillations, which, in our opinion, indicates a weak external loading of the samples (not more than $1 \%$ of the elastic limit).

The results of the experimental studies obtained in this paper do not contradict the literature data on the change in the physicochemical and mechanical properties of materials subject to various types of loads. The obtained experimental data should be used in the development of quick methods for diagnosing predefective states in depot conditions, simplifying the preliminary preparation of the studied object.

It should be noted that the results presented above were obtained under weak mechanical stresses and room temperatures. For the reliable determination of predefective states, it is planned to obtain similar experimental data, taking into account the existing values of the changes in loads and temperatures that arise under real operating conditions of the rolling stock.

\section{References}

1. R.Yu. Adadurov, Cars and rolling stock 4(44), 24 - 27 (2015)

2. G.S. Shelikhov, Magnetic particle inspection of products (Spectrum, Moscow, 2013)

3. V.F. Krivorudchenko, R.A. Akhmedzhanov, Modern methods of technical diagnostics and non-destructive examination of parts and units of rolling stock of railway transport (Marshrut, Moscow, 2005)

4. V.V. Zuev, L.N. Potselueva, Yu.D. Goncharov, Crystalloenergetics as a basis for evaluating the properties of solid materials (including magnesia cements) (Publishing house of the "Alfapol", SPb, 2006)

5. E. Clegg, W. G. Blevins, Wheel Impact Load Detector Experience on CN, American Railway Engineering Association Bulletin, 499-523 (1996)

6. J.M. Hart, N. Ahuja, C.P.L. Barkan, D.D. Davis, A Machine Vision System for Monitoring Railcar Health: Preliminary Results, Technology Digest: TD-04-008 (Association of American Railroads, Pueblo, Colorado, 2004)

7. J.R. Edwards, J.M. Hart, S. Todorovic, C.P.L. Barkan, N. Ahuja, Z. Chua, N. Kocher, J. Zeman, Proceedings of the International Heavy Haul Conference Specialist Technical Session - High Tech in Heavy Haul, Kiruna, 745-752 (2007)

8. G. den Buurman, European Railway Review 3, 80 - 86 (2005)

9. V.A. Gapanovich, Rail transport 10, 10-15 (2016)

10. V.V. Bolotin, Forecasting the resource of machines and structures (Mechanical Engineering, Moscow, 1984)

11. V.F. Terentev, S.A. Korableva, Fatigue of metals (Nauka, Moscow, 2015)

12. Yu.G. Matvienko, Models and criteria of the destruction mechanism (Fizmatlit, Moscow, 2006)

13. Yu.I. Matyash, Yu.M. Sosnovsky, A.A. Razhkovskii, E.M. Kondrikov, Omsk Scientific Bulletin. Ser. Mechanical Engineering and Engineering Science 4(154), 9-13 (2017)

14. V. Berzhansky, P. Maruschak, R. Biszak, I. Danilyuk, V. Vishnevsky, A. Prokopov, F. Pankov, N. Lugovskoy, News of TNTU 71-3, 272 - 278 (2015)

15. N.A. Kostenko, Prediction of reliability of transport vehicles (Mashinostroenie, Moscow, 1989) 
16. M.M. Zakirnichnaya, I.R. Kuzeev, V.K. Berdin, N.Yu. Kirillova, Oil and gas business, machines and devices 4-1, 207-214 (2006)

17. Yu.I. Matyash, Yu.M. Sosnovsky, E.M. Kondrikov, Patent №154462 Russian Federation, $G 01 \mathrm{~N} 3 / 38$, Laboratory installation for accelerated determination of the residual life of metallic ferromagnetic products taking into account the combined load (2015) 